\title{
Brief Overview
}

\section{META-ANALYSIS AS A RESEARCH DESIGN - STEPS DEMYSTIFIED FOR NOVICE MEDICAL RESEARCHERS: A BRIEF REVIEW}

\author{
Manoj Kumar L ${ }^{1 *}$ \\ ${ }^{1}$ Asst Professor, St Thomas College of Nursing, Kerala \\ *Corresponding Address: Asst Professor, St Thomas College of Nursing, Changanachery, Kerala. \\ Email:manojkumarlarson20@gmail.com
}

\begin{abstract}
Meta-analysis is a study design that assesses previous research quantitatively and arrives at a conclusion for a research question; that outcome or conclusion is considered strong enough to be kept in an apex position in the hierarchy of evidence. Meta-analysis is found to be a hurdle for most novice researchers due to complex statistical procedures and the availability of unbiased published literature globally. The lack of accurate theoretical background also adds to the aforementioned complexity. This paper tries to portray a simple, practical outline about meta-analysis, justification for using this design, other essential aspects concerned and methods to be used. Literature relevant to the topic published within ten years in prominent journals and websites has been reviewed thoroughly while writing this paper. Keywords used for literature search were; 'Meta-analysis', 'systematic review', 'Meta-analysis research design' and 'Meta-analysis framework'. The search was carried out after applying appropriate Boolean operators. Thoroughly and rigorously conducted meta-analysis is valid and has the potential of decision making in evidence-based medicine practice as it provides a more precise and cumulative estimate of the effect of a treatment/intervention, risk factor for a disease and or efficacy of drugs. The necessity to inculcate results or findings from numerous research studies makes it clear that meta-analysis as a research design is desirable in the field of medicine.
\end{abstract}

Keywords: Evidence-based medicine, Meta-analysis, Research design, systematic review.

\section{INTRODUCTION}

Abundance of research studies regarding a topic with divergent results is a common scenario while doing a literature search. It indicates the gross number of studies cumulated from various sources, especially in electronic format. These studies vary in study designs, sample size, population, and time frame, even though the exposure and outcome of interest will probably be the same. The aforementioned heterogeneity makes medical literature and associated knowledge base complex. This makes it difficult to draw a consensus from the results of certain research questions, leading to the complexity of conclusions. ${ }^{1}$ Systematic review and meta-analysis

Access the article online:

https://kjponline.com/index.php/kjp/article/view/293

DOI: https://doi.org/10.30834/KJP.34.2.2021.293

Received: 03/08/2021. Accepted: 20/09/2021.

Web publication: $13 / 10 / 2021$ pools the results of various studies and gives an unblemished conclusion.

The pyramid of evidence or synonymously portrayed hierarchy of evidence considers systematic review and meta-analysis (SR/MA) to have the highest evidence strength in practice. ${ }^{1}$ Well conducted SR/MA can be considered the first choice to make a conclusion feasible among the diverse studies focusing on a common outcome of interest. Complex statistical analysis and ess familiarity among novice researchers in the medical discipline make SR/MA a hurdle to pursue. But in this

Please cite this article as:. Manoj K L. Meta-analysis as a research design - steps demystified for novice medical researchers: a brief review. Kerala Journal of Psychiatry 2021; 34(2):170-179 
digital era, with the help of free software, complex statistical analysis is very easy to deal with. However, researchers must be aware of the basic concepts of $\mathrm{SR} / \mathrm{MA}$.

Systematic review is done based on a qualitative perspective, often followed by a meta-analysis - a strict quantitative paradigm. Perhaps, it is not mandatory to have meta-analyses in all systematic reviews, but all meta-analyses are performed on the background of a well-conducted systematic review. The popularity of $\mathrm{SR} / \mathrm{MA}$ is also increasing in the last few decades. ${ }^{2}$ The ultimate focus of meta-analyses is to determine the gravity of evidence regarding an intervention or treatment protocol or a drug. ${ }^{3}$ Simply meta-analysis is the statistical analysis of various study results to integrate them and make it more conclusive. ${ }^{4}$ The steps of systematic review and meta-analysis are more or less the same in all sense; meta-analysis is the statistical extension of the systematic review.

\section{Materials and methods}

An electronic search of the literature in the last decade (2011 to October 2021) highlighting meta-analysis, research designs, systematic review and their relationship with each other was conducted using the following databases: Google Scholar, Cochrane, Pub Med, Research Gate, and JSTOR. The inclusion criteria of the study involved (1) abstracts/full articles published in journals and (2) articles involving the theoretical background of meta-analysis as a research design. To avoid variations in definitions of the concept of meta-analysis, authors relied on understanding the concepts by the authors of the papers. The abstracts, original articles, and review articles published in languages other than English and with incomplete data were excluded. The keywords used for literature search included meta-analysis and or systematic review, metaanalysis research design and framework of metaanalysis. Overall, 35 relevant articles were utilized for this review. The review describes meta-analysis as a research design and steps of this design in a flawless manner.

\section{Steps in Conducting a Meta-analysis}

This article tries to demystify the steps of meta-analysis in a practical manner, making it comprehensible for young researchers to inculcate the theoretical background of the design, also taking into consideration standards established by various sources like Cochrane collaboration and the Institute of Medicine as well.

Steps can be listed as follows:

1. Formulating the research question

2. Locating the studies

3. Selection of studies and assessment of study quality

4. Data collection

5. Analysis

6. Presentation of results

These steps are listed after an extensive literature search regarding steps of meta-analysis in various sources and guidelines published by Cochrane collaboration; ${ }^{5}$ They can vary in different textbooks and published scientific articles.

\section{Formulating the research question}

Formulating research questions and objectives is a tedious task as in any kind of research study. Certain criteria's can be followed while formulating a research question; 'FINER' is a commonly used criterion (Research question should be - Feasible, Interesting, Novel, Ethical and Relevant). ${ }^{6}$ To meet these criteria, a logical and well-defined research question has to be formulated. Certain tools are available to assist this task, such as PICO, PICOS and SPIDER, which are used extensively in qualitative systematic reviews. ${ }^{7}$

Table 1 portrays three commonly used research question formulating tools. Among the three tools, PICO is widely used in systematic review and metaanalysis of randomized control trials.

Example 1: In adults with Covid-19 (Population), will administering an anti-viral drug $\mathrm{X}$ (Intervention) be effective in reducing the duration of symptoms (Outcome) as compared to steroids (Comparison)?

\section{Inclusion and exclusion criteria}

Once a research question is formulated, a set of inclusion and exclusion criteria need to be prepared to avoid bias in selecting studies. Study inclusion can be based on PICO format, date of publication and study design; in short, all studies we include must answer our research question clearly. Studies which are replicated, not available as full text, papers with abstracts only, which does not answer our research question, can be excluded. The eligibility and exclusion of study details can be portrayed using a flow chart, preferably the 
Table 1: Comparison of PICO, PICOS and SPIDER tools

\begin{tabular}{lll}
\hline \multicolumn{1}{c}{ PICO } & \multicolumn{1}{c}{ Research question formulating tools } \\
\hline Population & Population & \multicolumn{1}{c}{ SPIDER } \\
Intervention & Intervention & Sample \\
Comparison & Comparison & Phenomena of Interest \\
Outcome of Interest & Outcome of Interest & Design \\
& Study type & Evaluation \\
& & Research Type \\
\hline
\end{tabular}

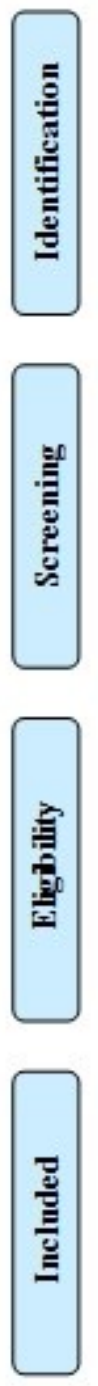

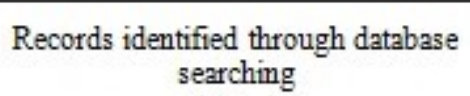

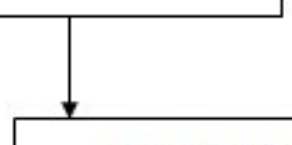

Records after duplicates removed $(\mathrm{n}=)$

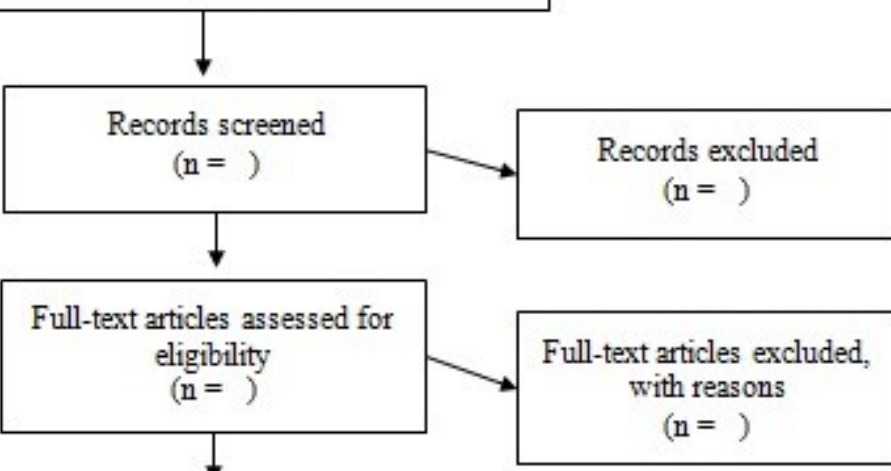

Studies included in qualitative synthesis $(n=)$

Studies included in quantitative synthesis (metaanalysis) $(n=)$

Figure 1: PRISMA Flowchart ${ }^{9}$ 
'Preferred Reporting of Items for Systematic Reviews and Meta-Analyses' (PRISMA) ${ }^{8}$ flow chart depicts the flow of information in systematic review followed by meta-analysis.

Figure 1 provides a flow chart that guides readers through how the researcher sorted out pertinent studies and came to a conclusion note.

For example, 1, inclusion criteria can be formulated as follows;

1. All randomized controlled trials evaluating the efficacy of drug X.

2. Including studies without any restriction based on country, date (unless set prior), gender, age, the language of publication and race of subjects.

Exclusion criteria can be;

1. Study of drug $\mathrm{X}$ in non-human subjects.

2. Study of drug $X$ in in-vitro studies.

3. Study with data overlapping and not reliably retrieved.

4. Studies published without full-text availability or abstract only papers.

5. Papers from conferences and editorials.

6. Case reports, case series papers and systematic reviews.

\section{Locating the studies}

\section{Search strategy and Databases}

An appropriate search strategy has to be developed. It is the crux of systematic review; search strategy is a cyclic process in which terms used for search are modified to get optimum results. Modification is done based on what has already been retrieved from previous searches; increasing the comprehensiveness of the search is always suggested. Details of search strategy have to be mentioned in the 'Methods' section of systematic review while reporting. Prominent databases have features like 'controlled vocabulary' to help identify randomized control trials and observational studies.

- Keywords and index terms - Keywords help get broader search results through journal titles, authors' names, and abstracts. Index terms help to narrow down and keep the focus on the searching process.
- Boolean Operators - This strategy helps broaden and narrow search aspects, commonly used are 'AND' (Helps to narrow down) and 'OR' (Helps to broaden by adding synonyms).

- Search Filters -Databases will have options called filters to narrow down your searches by applying article type, published year, language of subjects, gender of study subjects etc.

The aforementioned factors do play a vital role in creating search strategies. ${ }^{10}$ In doing SR, it is crucial to use both 'controlled vocabulary' and 'keywords/free text searching'. The controlled vocabulary used in Pub Med (A widely used database under the National Library of Medicine) is MeSH (Medical Subject Headings) which consists of descriptors and subheadings. A thesaurus is available to search for these terms and find definitions when we apply a term; it does have narrower and broader terms. ${ }^{6}$

\section{Search string for single and multiple concepts in a systematic review.}

If we search using MeSH in PubMed for Covid 19, it will give all possible studies if we have added the 'AND/OR' Boolean operator. For example, to search Alzheimer's as a single concept following search string can be used to avail optimum broad results;

("Alzheimer Disease"[mh] OR "alzheimer's"[tiab] OR "alzheimer"[tiab] OR ad[tiab] OR "alzheimers"[tiab] $O R$ "alzhiemer"[tiab] OR "alzhiemers"[tiab] OR "alzhiemer's"[tiab] OR "cognition disorders" mh] OR cognitive[tiab] OR cognition[tiab] OR "dementia"[mh:noexp] OR dementia[tiab])[10]. In PubMed search [mh] stands for Mesh headings, [mh:noexp] is used to turn off automatic explosion of MeSH headings and [tiab] denotes title or abstract.

\section{Documenting search strategies}

Search strategies formulated must be documented from electronic sources, hand searched journal articles, conference proceedings, and available unpublished articles. According to the PICO model, a research question will have multiple concepts, so always develop strategies for each concept and document. Table 2 gives a systematic way by which search strategies according to various concepts can be arranged for further reference. 
Table 2: Sample table for documenting search strategy ${ }^{11}$

\begin{tabular}{|c|c|c|c|}
\hline Key concepts & Concept 1 & Concept 2 & Concept 3 \\
\hline Free text terms searching & & & \\
Can do by phrase searching, truncations, common words etc. & & & \\
\hline $\begin{array}{c}\text { Controlled vocabulary terms / MeSH terms (PubMed search) } \\
\text { Headings, subheadings, descriptive terms etc. }\end{array}$ & & & \\
\hline
\end{tabular}

\section{Searching databases}

Minimum two databases have to be searched while conducting an SR. Certain guidelines such as 'The assessment of multiple systematic reviews' tool (AMSTAR) and checklist ${ }^{12}$ are available to evaluate the search and methods of SR.

PubMed, Scopus, Web of Science, Excerpta Medica Database (EMBASE), global health library (GHL), Von Hippel Lindau - Specific Clinical Database (VHL), Cochrane, Google Scholar, Clinicaltrials.gov, Meta Register of Controlled Trials (mRCT), The Cumulative Index of Nursing and Allied Health Literature (CINAHL), Conference papers index, PsycInfo, The Campbell Collaboration, Population Information Online (POPLINE), and System for Information on Grey Literature in Europe (SIGLE) are prominent databases which will cover almost all published articles from various disciplines. Researchers have to focus on appropriate databases to the research question and field of study, whether it's Medicine, Psychiatry, Nursing, Reproductive Health, Public Health, Epidemiology, etc.

\section{Grey literature}

It is a common term researchers see when they read about SR/MA; it's basically studies, literature and articles that are not published formally in any acclaimed platforms and databases, including indexed journals. Grey literature can be printed or electronic, including reports, conference proceedings, bibliographies, official documents, translations, theses, etc. Ageline, AltHealthWatch, Center for International Rehabilitation Research Information and Exchange (CIRRIE) database, Google Scholar, GreyNet, Hooked on Evidence, Meeting Abstracts via NLM Gateway,
National Technical Information Service (NTIS), New York Academy of Medicine Grey Literature Report, OAIster, OpenGrey aka OpenSIGLE (Europe), PsycExtra and Grey Literature for Dentistry are some of the databases to search for grey literature. ${ }^{13}$

\section{Selection of studies and assessment of study quality}

An amazingly large number of studies is available in electronic databases for the same research question but with different conclusions. RCTs are said to be the standard in establishing evidence, but many biases can creep in a meta-analysis, a quantitative synthesis of evidence from RCTs thereby will get affected by these biases. $^{14}$

Publication bias is defined as systematic flaws that arise when positive, significant results of clinical trials are more likely to get published. As a result, the studies we gather may have similar effects. Also, these studies are prone to get published in acclaimed English language journals and those indexed in Scopus or Medline. ${ }^{15,16}$ These results cannot be considered conclusive, and the data will also be misleading. A funnel plot test will be a proper measure to identify this bias. ${ }^{17}$ Detailed explanation of funnel plot test is out of the scope of this article.

This type of bias arises when studies with results that have significance are selectively reported. When a researcher performs MA, there is a probability of gathering such findings and ending in selection bias. ${ }^{18}$

Language Bias results when the selection of studies is based on restrictions to language. Mostly there is a tendency to search for articles published in the English language 
.${ }^{19}$ Attrition bias (loosing study participants, which are not reported in trials) and detection bias (bias arising in outcome assessment) are two other biases encountered less frequently in meta-analysis. ${ }^{20}$

\section{Appraisal of primary studies}

The Cochrane Collaboration risk of bias assessment tool ${ }^{21}$ for the appraisal of primary studies specifically for RCTs can be used. A series of questions in checklist form is used in this tool as follows:

- Adequate sequence generation done?

- Allocation concealment used?

- Blinding done?

- Concurrent therapies were similar?

- Incomplete outcome data addressed?

- Uniform and explicit outcome definitions?

- Free of selective outcome reporting?

Oxman and Guyatt index ${ }^{22}$, another tool with ten questions for appraisal of studies, can also be used in this concern. While performing an appraisal of a study, it may have adequate scores as per the grading checklist but, the study may not be having rigour in methodology. However,

there is an inclination by researchers to select such studies for MA/SR. A scoring system developed and validated by Jedad et al. can be used if grading is so particular for authors and researchers of SR/MA. ${ }^{23}$

Apart from the tools mentioned above; based on the study designs, plentiful tools are available; like NIH tool ${ }^{24}$ for observational and cross-sectional studies, SIGN critical appraisal checklists for the cohort study, ${ }^{25}$ ROBINS I tool for non-randomized control trials, ${ }^{26}$ CARE tool for case reports ${ }^{27}$, QUADAS tool for diagnostic purpose study designs ${ }^{28}$ and QUIPS tool for prognostic studies. ${ }^{29}$ It is always recommended to assess the quality of studies by two or three researchers independently to ensure the quality of studies and avoid bias.

\section{Data collection}

Collecting data from prominent databases is the cardinal part of a systematic review and later meta-analysis. As indicated above, researchers who have done data collection if getting engaged in further data analysis, such as; data cleaning, editing, and coding of data, will make the analysis process uncomplicated. ${ }^{30}$

The type of data to be extracted from selected studies is also an important aspect. Researchers will encounter different types of data when they start evaluating the results section of various RCTs. Data can be of dichotomous (or binary) data, continuous data, ordinal data (including measurement scales), counts and rates calculated from counting the number of events experienced by each individual, and time-to-event (typically survival) data. ${ }^{31}$ Readers can go through the Cochrane handbook or Institute of Medicine manual for systematic reviews for further details of data types, definitions, and explanations.

\section{Qualitative synthesis of collected data}

All collected studies should be taken through a primary evaluation; preparing an evidence table is paramount for this process to be at ease. An evidence table can be created to have a synthesis informally, and this will help to have an overall appraisal of selected studies before doing the quantitative analysis. Contents of this table may vary from researcher to researcher; still, the belowlisted data headings are minimally essential. With the help of a simple tabulation, as shown in table 3, it will be lucid to sort evidence gathered systematically and logically.

Table 3: Evidence table example

$\begin{array}{cccccccc}\text { Study } & \begin{array}{c}\text { Author } \\ \text { and Year }\end{array} & \begin{array}{c}\text { Sample } \\ \text { size }\end{array} & \begin{array}{c}\text { Quality } \\ \text { of Study }\end{array} & \text { Analysis } & \begin{array}{c}\text { Numbers } \\ \text { needed to } \\ \text { treat* }\end{array} & \text { Results } & \text { Comments } \\ & & & \end{array}$




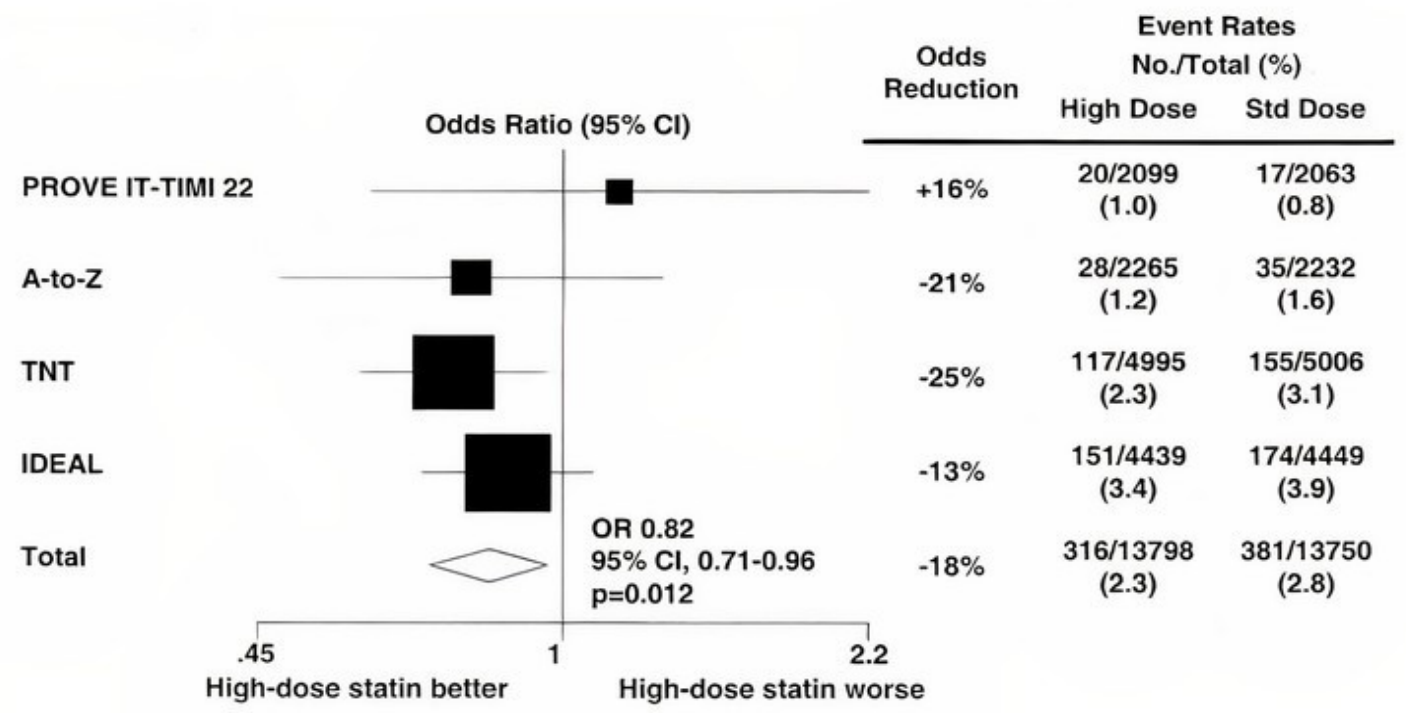

Figure 2: Example of an effect-Impact of statins in stroke prevention ${ }^{32}$

(Adapted from Canon et al., 2006)

\section{Analysis}

Statistical techniques to combine results of different studies to get an outcome estimate were introduced by Pearson in 1937. These statistical techniques are the crux of MA.

The core of meta-analysis or quantitative synthesis consists of two aspects as follows:

a. A pooled estimate and confidence interval for the 'effect' of treatment or intervention after combining all selected studies.

- The pooled estimate is simply the summary measure of all studies included in MA.

- For each study, we can assign certain weightage based on study peculiarities with the help of statistical formulae.

- Larger clinical trials are given more weightage.

- $95 \%$ confidence interval and $\mathrm{p}$-value for the pooled difference will also be identified.

The term effect has been used multiple times above; hence the researchers must know the concept of effect. Effect or effect size is a value that helps demonstrate the magnitude of treatment/intervention effect or sometimes the relationship between variables in a study. Effect size can be risk ratio, odds ratio, prevalence, correlation, mean difference, etc.
Meta-analysis incorporates information about the quality and reliability of the studies by weighing larger, better-reported studies more heavily. The effect size for individual studies is calculated initially. ${ }^{33}$

The above diagram is a forest plot (Blobbogram) representing results of MA conducted by Canon et al. Four studies are cumulated in this meta-analysis, and its weightage is represented by squares and the pooled estimated of effect is portrayed as a diamond. In the above example, the effect size is the odds ratio, and an odds ratio of 1.0 would mean no association with statins and prevention of stroke. Individual and pooled analyses demonstrate a significant $18 \%$ reduction of stroke with intensive statin therapy.

\section{Statistical methods:}

Depending on the type of effect sizes (Odds ratio, Risk Ratio, Correlation and so on), various statistical techniques are applied. Inverse-variance weighting, Mantel-Haenszel method, Peto method, Der Simonian and Laird method are commonly used measures to find pooled estimates. Among the above Mantel-Haenszel method and Peto method are widely used.

Readers can refer to Cochrane Handbook for Systematic Reviews of Interventions ${ }^{6}$ for detailed aspects of statistical analysis and explanations with examples. 


\section{b. Testing heterogeneity between included studies related to the effect of outcomes.}

Heterogeneity can arise due to different populations, study designs, the outcome of interest and treatments/interventions. This heterogeneity is identified statistically by a chi-squared test with degrees of freedom number of studies minus one.

\section{Fixed effect versus Random effects Model}

In the fixed-effects model, the basic assumption is that the effect size of all studies is identical and, if it's varying, it is due to sampling error. On the contrary, the random effects model assumes that the mean value of effects needs to be determined and that the goal is to analyze variance among treatments or interventions. Both models have their assumptions; however, many studies have reported that there are negligible differences between models in terms of effect size. ${ }^{21}$

\section{Software to perform a meta-analysis}

Stata/WinBUGS, R/OpenBUGS, MIX, CMA, RevMan and Meta-Analyst are the prominent software available now; among these, RevMan and Meta-Analyst are free software. ${ }^{34,35}$ They help in statistical analysis, funnel plot and forest plot generation.

\section{Presentation of results}

Data extraction and the results of meta-analysis results should be presented clearly to make the findings evidently visible to readers. A table should be prepared to outline the features of the studies, such as the characteristics of subjects, study design, sample size, and intervention, including the dosage of drugs and duration of any drugs or given intervention. The Forest plot is the graphical representation of MA. A square represents the point estimate for the effect size, and a horizontal line represents the confidence interval for each study. The size of the square represents the weight of each study. ${ }^{36}$

\section{Reporting Meta-analysis}

It is always recommended to write SR/MA in the standard sections: Introduction, Materials and methods, Results and Discussion. A PRISMA flow diagram should be prepared to report the flow of studies. PRISMA $^{37}$ and MOOSE $^{38}$ guidelines are commonly used for reporting SR \& MA; among the two, PRISMA is the most preferred guideline worldwide.

\section{Conclusion}

$\mathrm{SR} / \mathrm{MA}$ is not that favourable and fascinating research design for young researchers. However, the popularity is getting emaciated in the last two decades. The theoretical background of SR/MA looks easy, but the statistical perspectives and proper analysis make it hard for young researchers to pursue. However, SR/MA provides the greatest strength to available evidence in the field of Medicine and Public Health. Hence, novice researchers must grasp the powerful yet inflexible research designs.

\section{Conflict of interest}

The author declares that there is no conflict of interest.

\section{Ethical considerations}

None significant

\section{Funding}

The author received no funding.

\section{References}

1. Bello A, Wiebe N, Garg A, Tonelli M. Evidence-based decision-making 2: Systematic reviews and metaanalysis. Methods Mol Biol. 2015;1281:397-416. doi: 10.1007/978-1-4939-2428-8_24. PMID: 25694324.

2. Burns PB, Rohrich RJ, Chung KC. The Levels of Evidence and their role in Evidence-Based Medicine. Plast Reconstr Surg. 2011 Jul;128(1):305-10.

3. Greco T, Zangrillo A, Biondi-Zoccai G, Landoni G. Meta-analysis: pitfalls and hints. Heart Lung Vessel. 2013; 5(4):219-25.

4. Haidich AB. Meta-analysis in medical research. Hippokratia. 2010 Dec; 14(Suppl 1):29-37.

5. Glass GV. Primary, secondary and meta-analysis of research. Educational Researcher. 1976;5:3-8.

6. Higgins JPT, Green S. Cochrane Handbook for Systematic Reviews of Interventions Version 5.1.0 [updated March 2011]. 2011.

7. Aslam S, Emmanuel P. Formulating a researchable question: A critical step for facilitating good clinical research. Indian $\mathrm{J}$ Sex Transm Dis AIDS. 2010;31(1):47-50.

8. Methley AM, Campbell S, Chew-Graham C, McNally R, Cheraghi-Sohi S. PICO, PICOS and SPIDER: a comparison study of specificity and sensitivity in three search tools for qualitative systematic reviews. BMC Health Serv Res [Internet]. 2014 Nov 21 [cited 2020 Sep 12];14. Available from: https://www.ncbi.nlm.nih.gov/pmc/articles/PMC4310146/ 
9. Moher D, Liberati A, Tetzlaff J, Altman DG; PRISMA Group. Preferred reporting items for systematic reviews and meta-analyses: the PRISMA statement. PLoS Med. 2009;6(7):e1000097. doi: 10.1371/journal.pmed.1000097.

10. Library THS. Research Guides: Systematic Reviews: Creating a Search Strategy [Internet]. [Cited 2020 Sep 13]. Available from: https: / guides.lib.umich.edu/c.php?g=283340\&p=2126706

11. Aromataris, Edoardo\&Riitano, Dagmara. (2014). Constructing a search strategy and searching for evidence. A guide to the literature search for a systematic review. The American journal of nursing. 114. 49-56.

12. Shea BJ, Reeves BC, Wells G, Thuku M, Hamel C, Moran J, Moher D et al. AMSTAR 2: a critical appraisal tool for systematic reviews that include randomized or non-randomized studies of healthcare interventions, or both. BMJ. 2017 ;358:j4008. doi: 10.1136/bmj.j4008.

13. Alberani V, De Castro Pietrangeli P, Mazza AM. The use of grey literature in health sciences: a preliminary survey. Bull Med Libr Assoc. 1990 Oct;78(4):358-63.

14. Stegenga J. Is meta-analysis the platinum standard of evidence? Stud Hist Philos Biol Biomed Sci. 2011 Dec;42(4):497-507. doi: 10.1016/j.shpsc.2011.07.003.

15. Peter Jüni, Franziska Holenstein, Jonathan Sterne, Christopher Bartlett, Matthias Egger, Direction and impact of language bias in meta-analyses of controlled trials: empirical study, International Journal of Epidemiology, Volume 31, Issue 1, February 2002: 115123.

16. Dubben H-H, Beck-Bornholdt H-P. Systematic review of publication bias in studies on publication bias. BMJ. 2005 Aug 20;331(7514):433-4.

17. Egger M, Davey Smith G, Schneider M, Minder C. Bias in meta-analysis detected by a simple, graphical test. BMJ. 1997 Sep 13;315(7109):629-34.

18. Williamson, P. R. et al. (2005) 'Outcome selection bias in meta-analysis', Statistical Methods in Medical Research, 14(5), pp. 515-524.

19. Zlowodzki M, Poolman RW, Kerkhoffs GM, Tornetta $\mathrm{P}$, Bhandari M; International Evidence-Based Orthopedic Surgery Working Group. How to interpret a meta-analysis and judge its value as a guide for clinical practice. Acta Orthop. 2007 Oct;78(5):598-609. doi: 10.1080/17453670710014284. PMID: 17966018.

20. Higgins JPT, Thomas J, Chandler J, Cumpston M, Li T, Page MJ, Welch VA (editors). Cochrane Handbook for Systematic Reviews of Interventions version 6.0 (updated July 2019).

21. Higgins JPT, Green S. Cochrane Handbook for Systematic Reviews of Interventions. 2008: The Cochrane Collaboration, Oxford.
22. Oxman AD, Guyatt GH. Validation of an index of the quality of review articles. J Clin Epidemiol 1991; 44: 1271-8.

23. Jadad AR, Moore RA, Carroll D, et al. Assessing the quality of reports of randomized clinical trials: is blinding necessary? Controlled Clinical Trials 1996;17[1]:1-12.

24. Study Quality Assessment Tools | NHLBI, NIH [Internet]. [cited 2020 Sep 15]. Available from: https://www.nhlbi.nih.gov/health-topics/studyquality-assessment-tools.

25. Glynn L. A critical appraisal tool for library and information research. Cleyle S, editor. Library Hi Tech. 2006 Jul;24(3):387-99.

26. Sterne JAC, Hernán MA, Reeves BC, Savović J, Berkman ND, Viswanathan M, et al. ROBINS-I: a tool for assessing risk of bias in non-randomized studies of interventions. BMJ. 2016;355.

27. CARE Checklist [Internet]. CARE Case Report Guidelines. [cited 2020 Sep 15]. Available from: https://www.care-statement.org/checklist.

28. Whiting P, Rutjes AW, Reitsma JB, Bossuyt PM, Kleijnen J. The development of QUADAS: a tool for the quality assessment of studies of diagnostic accuracy included in systematic reviews. BMC Medical Research Methodology. 2003 Nov 10;3(1):25.

29. Hayden JA, van der Windt DA, Cartwright JL, Côté P, Bombardier C. Assessing bias in studies of prognostic factors. Ann Intern Med. 2013;158(4):280-286.

30. Pedder H, Sarri G, Keeney E, Nunes V, Dias S. Data extraction for complex meta-analysis guide. Syst Rev [Internet]. 2016 [cited 2020 Sep 15];5. Available from: https://www.ncbi.nlm.nih.gov/pmc/articles/PMC5154138/

31. Higgins JPT, Li T, Deeks JJ (editors). Chapter 6: Choosing effect measures and computing estimates of effect. In: Higgins JPT, Thomas J, Chandler J, Cumpston M, Li T, Page MJ, Welch VA (editors). Cochrane Handbook for Systematic Reviews of Interventions version 6.0 (updated July 2019). Cochrane, 2019.

32. Cannon CP, Steinberg BA, Murphy SA, Mega JL, Braunwald E. Meta-Analysis of Cardiovascular Outcomes Trials Comparing Intensive Versus Moderate Statin Therapy. Journal of the American College of Cardiology. 2006 Aug;48(3):438-45.

33. Borenstein, M., Hedges, L. V., Higgins, J. P. T., \& Rothstein, H. (2009). Introduction to meta-analysis. Chichester, U.K: John Wiley \& Sons.

34. Wallace, B.C., Schmid, C.H., Lau, J. et al. MetaAnalyst: software for meta-analysis of binary, 
continuous and diagnostic data. BMC Med Res Methodol9, 80 (2009).

35. Wang J, Leeflang M. Recommended software/packages for meta-analysis of diagnostic accuracy. J Lab Precis Med 2019;4:22.

36. Chalmers TC, Buyse M. Meta-analysis. In: Chalmers TC, Blum A, Buyse M, et al., editors. Data Analysis for Clinical Medicine: The Quantitative Approach to Patient Care. Rome, Italy: International University Press; 1988. pp. 75-84
37. Liberati Alessandro, Altman Douglas G, Tetzlaff Jennifer, Mulrow Cynthia, Gøtzsche Peter C, Ioannidis John P A et al. The PRISMA statement for reporting systematic reviews and meta-analyses of studies that evaluate healthcare interventions: explanation and elaboration BMJ 2009; 339:b2700

38. Stroup DF, Berlin JA, Morton SC, et al. Meta-analysis of Observational Studies in Epidemiology: A Proposal for Reporting. JAMA. 2000;283(15):2008-2012. doi:10.1001/jama.283.15.2008. 